DO-TH-99-21

OHSTPY-HEP-T-99-026

\title{
Searching for Rephase-Invariant CP- and CPT-violating Observables in Meson Decays
}

\author{
K.C. Chou ${ }^{a}$, W.F. Palmer ${ }^{b}$, E.A. Paschos ${ }^{c}$ and Y. L. Wu ${ }^{a, c}$ \\ $a$ : Institute of Theoretical Physics, Chinese Academy of Sciences \\ Beijing 100080, China \\ $b$ : Department of Physics, Ohio-State University \\ Columbus, OH 43210, USA \\ $c$ : Institut für Physik, Universität Dortmund \\ D-44221 Dortmund, Germany \\ palmer@mps.ohio-state.edu \\ paschos@hal1.physik.uni-dortmund.de \\ ylwu@itp.ac.cn
}

\begin{abstract}
We present a general model-independent and rephase-invariant formalism that cleanly relates $\mathrm{CP}$ and $\mathrm{CPT}$ noninvariant observables to the fundamental parameters. Different types of CP and CPT violations in the $K^{0}-, B^{0}-, B_{s}^{0}$ and $D^{0}$-systems are explicitly defined. Their importance for interpreting experimental measurements of $\mathrm{CP}$ and $\mathrm{CPT}$ violations is emphasized. In particular, we show that the timedependent measurements allow one to extract a clean signature of CPT violation.
\end{abstract}

PACS numbers: 11.30.Er, 13.25. $+\mathrm{m}$ 


\section{Introduction}

For the discrete symmetries of nature, violations have been observed for $\mathrm{C}, \mathrm{P}$ and the combined CP symmetries 11, 2, 3, 4, 5]. In fact two types of $\mathrm{CP}$ violation have now been established in the $K$-meson system. It remains an active problem of research to observe $\mathrm{CP}$ asymmetries in heavier mesons. In addition there is new interest in investigations of properties of the CPT symmetry [6]. Up to now, there are only bounds on CPT-violating parameters[7], which are sensitive to the magnitude of amplitudes, but tests of the relative phases have not yet been carried out.

In this article we present tests of CPT and CP, separately, and discuss which measurements distinguish between the various symmetry breaking terms. In addition, we derive formulae which are manifestly invariant under rephasing of the original mesonic states. The hope is to call attention to several measurements which will be accessible to experiments in the future.

Our paper is organized as follows: In section 2, we present a complete set of parameters characterizing $\mathrm{CP}, \mathrm{T}$ and $\mathrm{CPT}$ nonconservation arising from the mass matrix, i.e., the so-called indirect CP-, T- and CPT-violation. A set of direct CP-, T- and CPT-violating parameters originating from the decay amplitudes are defined in section 3 . In section 4 , we defind all possible independent observables and relate them directly to fundamental parameters which are manifestly rephasing invariant and can be applied to all meson decays. The various types of $\mathrm{CP}$ and $\mathrm{CPT}$ violation are classified, indicating how one can extract purely CPT or CP violating effects. In section 5, we investigate in detail the time evolution of mesonic decays and introduce several time-dependent CP- and CPTasymmetries which allow one to measure separately the indirect CPT- and CP-violating observables as well as direct CPT- and CP-violating observables. In particular, we show how one can extract a clean signature of CPT violation from asymmetries in neutral meson decays. In section 6, we apply the general formalism to the semileptonic and nonleptonic $\mathrm{K}$-meson decays and show how many rephasing invariant $\mathrm{CP}$ and $\mathrm{CPT}$ observables can be extracted separately. Our conclusions are presented in the last section.

\section{CP- and CPT-violating Parameters in Mass Ma- trix}

Let $M^{0}$ be the neutral meson (which can be $K^{0}$ or $D^{0}$ or $B^{0}$ or $B_{s}^{0}$ ) and $\bar{M}^{0}$ its antiparticle. The evolution of $M^{0}$ and $\bar{M}^{0}$ states is dictated by

$$
\frac{d}{d t}\left(\begin{array}{c}
M^{0} \\
\bar{M}^{0}
\end{array}\right)=-i\left(\begin{array}{ll}
H_{11} & H_{12} \\
H_{21} & H_{22}
\end{array}\right)\left(\begin{array}{c}
M^{0} \\
\bar{M}^{0}
\end{array}\right)
$$

with $H_{i j}=M_{i j}-i \Gamma_{i j} / 2$ the matrix elements, and $M_{i j}, \Gamma_{i j}$ being the dispersive and absorptive parts, respectively. 
The eigenvalues of the Hamiltonian are

$$
\begin{aligned}
& H_{1}=H_{11}-\sqrt{H_{12} H_{21}} \frac{1-\Delta_{M}}{1+\Delta_{M}}, \\
& H_{2}=H_{22}+\sqrt{H_{12} H_{21}} \frac{1-\Delta_{M}}{1+\Delta_{M}},
\end{aligned}
$$

with

$$
\frac{1-\Delta_{M}}{1+\Delta_{M}}=\left[1+\frac{\delta_{M}^{2}}{2}-\delta_{M} \sqrt{1+\frac{\delta_{M}^{2}}{4}}\right]^{1 / 2}, \quad \text { and } \quad \delta_{M}=\frac{H_{22}-H_{11}}{\sqrt{H_{12} H_{21}}}
$$

We note already that $\delta_{M}$ is invariant under rephasing of the states $M^{0}$ and $\bar{M}^{0}$. The eigenfunctions of the Hamiltonian define the physical states. Following Bell and Steinberger 88, $M^{0}$ and $\bar{M}^{0}$ mix with each other and form two physical mass eigenstates

$$
M_{1}=p_{S}\left|M^{0}>+q_{S}\right| \bar{M}^{0}>, \quad M_{2}=p_{L}\left|M^{0}>-q_{L}\right| \bar{M}^{0}>
$$

with normalization $\left|p_{S}\right|^{2}+\left|q_{S}\right|^{2}=\left|p_{L}\right|^{2}+\left|q_{L}\right|^{2}=1$. The coeficients are given by

$$
\begin{aligned}
\frac{q_{S}}{p_{S}} & =\frac{q}{p} \frac{1+\Delta_{M}}{1-\Delta_{M}} \equiv \frac{1-\epsilon_{S}}{1+\epsilon_{S}}, \quad \frac{q_{L}}{p_{L}}=\frac{q}{p} \frac{1-\Delta_{M}}{1+\Delta_{M}} \equiv \frac{1-\epsilon_{L}}{1+\epsilon_{L}} \\
\frac{q}{p} & =\sqrt{\frac{H_{21}}{H_{12}}} \equiv \frac{1-\epsilon_{M}}{1+\epsilon_{M}}
\end{aligned}
$$

We have also introduced the paramters $\epsilon_{S, L, M}$ following ref.[9]. In the CPT conserving case they reduce to the known parameter $\epsilon_{M}$. Thus we have a complete description of the physical states in terms of the mass matrix, and the time evolution is determined by the eigenvalues:

$$
H_{1}=M_{1}-i \Gamma_{1} / 2 ; \quad H_{2}=M_{2}-i \Gamma_{2} / 2
$$

and is given simply by

$$
M_{1} \rightarrow e^{-i H_{1} t} M_{1} ; \quad M_{2} \rightarrow e^{-i H_{2} t} M_{2}
$$

We discuss next several properties related to the symmetries of the system. The parameters $\delta_{M}$ and $|q / p|$ are rephasing invariant and so are also other parameters defined in terms of them. CPT invariance requires $M_{11}=M_{22}$ and $\Gamma_{11}=\Gamma_{22}$, and implies that $\delta_{M}=0$. Thus the difference between $q_{S} / p_{S}$ and $q_{L} / p_{L}$ represents a signal of CPT violation. In other words, $\Delta_{M}$ different from zero indicates CPT violation.

$\mathrm{CP}$ invariance requires the dispersive and absorptive parts of $H_{12}$ and $H_{21}$ to be, respectively, equal and implies $q / p=1$. Also if $\mathrm{T}$ invariance holds, then independently of CPT symmetry, the dispersive and absorptive parts of $H_{12}$ and $H_{21}$ must be equal up to a total relative common phase, implying $|q / p|=1$. Therefore a $\operatorname{Re}_{M}$ different from zero describes $\mathrm{CP}$ and $\mathrm{T}$ nonconservation and can be present even when CPT is conserved. Finally, two parameters, $\epsilon_{M}$ describing $\mathrm{CP}$ violation with $\mathrm{T}$ nonconservation and $\Delta_{M}$ characterizing CPT violation with $\mathrm{CP}$ nonconservation, are related to $\epsilon_{S}$ and $\epsilon_{L}$ via

$$
\epsilon_{S}=\frac{\epsilon_{M}-\Delta_{M}}{1-\epsilon_{M} \Delta_{M}} ; \quad \epsilon_{L}=\frac{\epsilon_{M}+\Delta_{M}}{1+\epsilon_{M} \Delta_{M}}
$$


and reduce to those given in [9] when neglecting the quadratic term $\epsilon_{M} \Delta_{M}$. This is a complete set of parameters describing $\mathrm{CP}, \mathrm{T}$ and $\mathrm{CPT}$ nonconservation which originates in the mass matrix (indirect). In the next section we discuss additional parameters originating in the decay amplitudes (direct) as well as from the mixing between mass matrix and decay amplitudes (mixed-induced).

\section{CP- and CPT-violating Parameters in Decay Am- plitudes}

Let $H_{\text {eff }}$ be the effective Hamiltonian which contains CPT-even $H_{\text {eff }}^{(+)}$and CPT-odd $H_{e f f}^{(-)}$ parts, i.e.,

$$
H_{e f f}=H_{e f f}^{(+)}+H_{e f f}^{(-)}
$$

with

$$
(C P T) H_{e f f}^{( \pm)}(C P T)^{-1}= \pm H_{e f f}^{( \pm)}
$$

Let $f$ denote the final state of the decay and $\bar{f}$ its charge conjugate state. The decay amplitudes of $M^{0}$ are defined as

$$
\begin{aligned}
g & \equiv<f\left|H_{e f f}\right| M^{0}>=\sum_{i}\left(A_{i}+B_{i}\right) e^{i \delta_{i}} \equiv \sum_{i}\left(\left|A_{i}\right| e^{i \phi_{i}^{A}}+\left|B_{i}\right| e^{i \phi_{i}^{B}}\right) e^{i \delta_{i}} \\
\bar{h} & \equiv<\bar{f}\left|H_{e f f}\right| M^{0}>=\sum_{i}\left(C_{i}+D_{i}\right) e^{i \delta_{i}} \equiv \sum_{i}\left(\left|C_{i}\right| e^{i \phi_{i}^{C}}+\left|D_{i}\right| e^{i \phi_{i}^{D}}\right) e^{i \delta_{i}}
\end{aligned}
$$

with $A_{i}$ and $C_{i}$ being CPT-conserving amplitudes

$$
<f\left|H_{e f f}^{(+)}\right| M^{0}>\equiv \sum_{i} A_{i} e^{i \delta_{i}}, \quad<\bar{f}\left|H_{e f f}^{(+)}\right| M^{0}>\equiv \sum_{i} C_{i} e^{i \delta_{i}}
$$

and $B_{i}$ and $D_{i}$ being CPT-violating amplitudes

$$
<f\left|H_{\text {eff }}^{(-)}\right| M^{0}>\equiv \sum_{i} B_{i} e^{i \delta_{i}}, \quad<\bar{f}\left|H_{e f f}^{(-)}\right| M^{0}>\equiv \sum_{i} D_{i} e^{i \delta_{i}}
$$

Here we have used the notation of ref.[10] for the amplitude $g$, and have introduced a new amplitude $\bar{h}$. The second amplitude is absent when one considers only K-meson decays and neglects possible violation of $\Delta S=\Delta Q$ rule as was the case in ref. [10]. This is because the K-meson decays obey $\Delta S=\Delta Q$ rule via weak interactions of the standard model. The reason is simple since the strange quark can only decay to the up quark. In the case of $B$-, $B_{s^{-}}$and $D$-meson systems both amplitudes $g$ and $\bar{h}$ exist via the $W$-boson exchange of weak interactions since both $b$-quark and $c$-quark will have two different transitions due to CKM quark mixings, i.e., $b \rightarrow c, u$ and $c \rightarrow s, d$ (for explicit decay modes see the classification for the processes given in section 5$)$. $\phi_{i}^{I}(I=A, B, C, D)$ are weak phases and $\delta_{i}$ are strong phases from final state interactions. The subscrpts $i=1,2, \cdots$ denote various strong interacting final states, such as the different isospin states. For CP transformation, we adopt the phase convention

$$
C P\left|M^{0}>=\right| \bar{M}^{0}>, \quad C P\left|\bar{M}^{0}>=\right| M^{0}>,
$$


It is then not difficult to show that the decay amplitudes of the charge conjugate meson $\bar{M}^{0}$ have the following form

$$
\begin{aligned}
& \bar{g} \equiv<\bar{f}\left|H_{e f f}\right| \bar{M}^{0}>=\sum_{i}\left(A_{i}^{*}-B_{i}^{*}\right) e^{i \delta_{i}} \equiv \sum_{i}\left(\left|A_{i}\right| e^{-i \phi_{i}^{A}}-\left|B_{i}\right| e^{-i \phi_{i}^{B}}\right) e^{i \delta_{i}} \\
& h \equiv<f\left|H_{e f f}\right| \bar{M}^{0}>=\sum_{i}\left(C_{i}^{*}-D_{i}^{*}\right) e^{i \delta_{i}} \equiv \sum_{i}\left(\left|C_{i}\right| e^{-i \phi_{i}^{C}}-\left|D_{i}\right| e^{-i \phi_{i}^{D}}\right) e^{i \delta_{i}} .
\end{aligned}
$$

In analogy to the indirect $\mathrm{CP}$ - and CPT-violating parameters $\epsilon_{S, L, M}$ from mass matrix, we define now parameters containing direct $\mathrm{CP}$ and $\mathrm{CPT}$ violations

$$
\varepsilon_{M}^{\prime} \equiv \frac{1-h / g}{1+h / g}, \quad \bar{\varepsilon}_{M}^{\prime} \equiv \frac{1-\bar{g} / \bar{h}}{1+\bar{g} / \bar{h}} ; \quad \varepsilon_{M}^{\prime \prime} \equiv \frac{1-\bar{g} / g}{1+\bar{g} / g}, \quad \bar{\varepsilon}_{M}^{\prime \prime} \equiv \frac{1-h / \bar{h}}{1+h / \bar{h}}
$$

For final states which are CP conjugate, i.e., $|\bar{f}>=C P| f>=\mid f>$, the relations $h=\bar{g}$ and $\bar{h}=g$ hold, and thus the four parameters are reduced to two independent ones: $\varepsilon_{M}^{\prime}=\varepsilon_{M}^{\prime \prime}$ and $\bar{\varepsilon}_{M}^{\prime}=\bar{\varepsilon}_{M}^{\prime \prime}$.

The symmetry properties of the amplitudes are as follows. If CP is conserved, independently of CPT symmetry, one has $\bar{g} / g=1$ and $h / \bar{h}=1$, which implies

$$
A_{i}=A_{i}^{*}, \quad C_{i}=C_{i}^{*}, \quad B_{i}=-B_{i}^{*}, \quad D_{i}=-D_{i}^{*}
$$

in other words:

$$
\phi_{i}^{A}=\phi_{i}^{C}=0, \quad \phi_{i}^{B}=\phi_{i}^{D}=\pi / 2,
$$

namely, $A_{i}$ and $C_{i}$ are real, while $B_{i}$ and $D_{i}$ are imaginary.

Similarly $\mathrm{T}$ invariance exchanges the initial and final states and implies, independently of CPT symmetry,

$$
A_{i}=A_{i}^{*}, \quad C_{i}=C_{i}^{*}, \quad B_{i}=B_{i}^{*}, \quad D_{i}=D_{i}^{*}
$$

or

$$
\phi_{i}^{A}=\phi_{i}^{C}=0, \quad \phi_{i}^{B}=\phi_{i}^{D}=0,
$$

namely, all the amplitudes must be real. Finally, conservation of CPT requires $B_{i}=0$ and $D_{i}=0$. We summarize the results for the amplitudes in Table 1 .

Table 1.

\begin{tabular}{|c|cc|cc|}
\hline & \multicolumn{2}{|c|}{ CPT-conservation } & \multicolumn{2}{c|}{ CPT-Violation } \\
\hline CP-conservation & $A_{i}=A_{i}^{*}$ & $C_{i}=C_{i}^{*}$ & $B_{i}=-B_{i}^{*}$ & $D_{i}=-D_{i}^{*} \quad$ imply T-violation \\
& & & \\
T-conservation & $A_{i}=A_{i}^{*}$ & $C_{i}=C_{i}^{*}$ & $B_{i}=B_{i}^{*}$ & $D_{i}=D_{i}^{*} \quad$ imply CP-violation \\
& $\mathrm{CP} \& \mathrm{~T}$ conservation & & & \\
\hline
\end{tabular}


Reading across the first row of the table we have the conditions for CP conservation, with $\mathrm{T}$ conservation (first column) and without T-conservation (second column). The relations $B_{i}=-B_{i}^{*}$ and $D_{i}=-D_{i}^{*}$ imply T-violation in the presence of CP conservation. The second row of the table gives the conditions when $\mathrm{T}$ is conserved, with $\mathrm{CP}$ conservation (first column) or without CP conservation (second column). This is a complete set of amplitude with the $C_{i}$ and $D_{i}$ amplitudes introduced for the first time here. As a consequence, two more CP- and CPT-violating parameters $\varepsilon_{M}$ and $\bar{\varepsilon}_{M}$ in eq. (16) are needed.

In summary of this section, we have the following conclusions. Values for $\operatorname{Re}_{M}^{\prime \prime}$ and $\operatorname{Re} \bar{\varepsilon}_{M}^{\prime \prime}$ different from zero describe CP nonconservation independently of $T$ and CPT symmetries. The presence of $B_{i}^{\prime} s$ and $D_{i}^{\prime} s$ indicate simultaneous nonconservation of: CPT and either of $C P$ or $T$. Zero $\varepsilon_{M}^{\prime \prime}$ and $\bar{\varepsilon}_{M}^{\prime \prime}$ with nonzero $\operatorname{Im} \varepsilon_{M}^{\prime}$ and $\operatorname{Im} \bar{\varepsilon}_{M}^{\prime}$ implies $T$ nonconservation. Finally, zero $B_{i}$ and $D_{i}$, and complex $A_{i}$ and $C_{i}$ signal $C P T$ conservation with $C P$ and $T$ violations. Note that the latter case is more difficult to establish experimentally since it requires the observation of a relative phase between two amplitudes distinguished with the help of specific quantum numbers. This was the case with the $\epsilon^{\prime} / \epsilon$ parameter in K-meson decays.

\section{Rephase Invariant CP- and CPT-violating Observ- ables}

The $\varepsilon$-type parameters defined in eqs.(5) and (16) can not be related to physical observables since they are not rephasing invariant. Let us introduce CP- and CPT-violating observables by considering the ratio,

$$
\hat{\eta}_{f} \equiv \frac{q_{S}}{q_{L}} \frac{<f\left|H_{e f f}\right| M_{2}>}{<f\left|H_{e f f}\right| M_{1}>}=\frac{q_{S}}{q_{L}} \frac{p_{L}}{p_{S}} \frac{1-r_{f}^{L}}{1+r_{f}^{S}}
$$

which enters to the time evolution of the decay amplitudes (see eqs. 27 and 28). The parameters $q_{S, L}$ and $p_{S, L}$ were defined in section 2 , and we also introduce the notation

$$
r_{f}^{S}=\left(q_{S} / p_{S}\right)(h / g)
$$

with a similar definition for $r_{f}^{L}$. Note that the factor $q_{S} / q_{L}$ is necessary for the normalization and also rephase invariance, which has not been always included in the literature. In the CPT-conserving case [11] this factor is equal to unity. One can simply see from the definitions in eqs.(3)-(5) that $\hat{\eta}_{f}$ is rephasing invariant. The factor $q_{S} p_{L} / p_{S} q_{L}=\left(1+\Delta_{M}\right)^{2} /\left(1-\Delta_{M}\right)^{2}$ is rephase-invariant since $\Delta_{M}$ has this property. The ratios $r_{f}^{L, S}=\left(q_{L, S} / p_{L, S}\right)(h / g)$ are also rephase-invariant. To see that, let us make a phase redefinition $\left|M^{0}>\rightarrow e^{i \phi}\right| M^{0}>$, then $\left|\bar{M}^{0}>\rightarrow e^{-i \phi}\right| \bar{M}^{0}>, H_{12} \rightarrow e^{-2 i \phi} H_{12}$ and $H_{21} \rightarrow$ $e^{2 i \phi} H_{21}$, as well as $h \rightarrow e^{-i \phi} h$ and $g \rightarrow e^{i \phi} g$, thus $\left(q_{S} / p_{S}, q_{L} / p_{L}\right) \rightarrow e^{2 i \phi}\left(q_{S} / p_{S}, q_{L} / p_{L}\right)$ and $h / g \rightarrow e^{-2 i \phi} h / g$, which makes $r_{f}^{L, S}=\left(q_{L, S} / p_{L, S}\right)(h / g)$ manifestly rephase-invariant. 
It is seen that the rephase-invariant quantities $r_{f}^{L, S}$ and $\hat{\eta}_{f}$ are given by the product of complex parameters arising from the mass mixing $\left(q_{L, S} / p_{L, S}\right)$ and from amplitudes $(h / g)$. To separately define the rephase-invariant CP- and CPT-violating observables originating from the mass mixing and from the amplitudes, some algebra is neccesary円, but it is not difficult to show that $\hat{\eta}_{f}$ can be rewritten as

$$
\hat{\eta}_{f} \equiv \frac{1}{1-\eta_{\Delta}}\left[\eta_{\Delta}+\frac{a_{\epsilon_{S}}+\hat{a}_{\epsilon^{\prime}}+i \hat{a}_{\epsilon_{S}+\epsilon^{\prime}}}{2+a_{\epsilon_{S}} \hat{a}_{\epsilon^{\prime}}+\hat{a}_{\epsilon_{S} \epsilon^{\prime}}}\right]
$$

where we have used the definitions

$$
\begin{aligned}
a_{\epsilon_{S}} & =\frac{1-\left|\frac{q_{S}}{p_{S}}\right|^{2}}{1+\left|\frac{q_{S}}{p_{S}}\right|^{2}}=\frac{2 R e \epsilon_{S}}{1+\left|\epsilon_{S}\right|^{2}}=\frac{a_{\epsilon}-a_{\Delta}}{1-a_{\epsilon} a_{\Delta}}, \\
a_{\epsilon_{L}} & =\frac{1-\left|\frac{q_{L}}{p_{L}}\right|^{2}}{1+\left|\frac{q_{L}}{p_{L}}\right|^{2}}=\frac{2 \operatorname{Re} \epsilon_{L}}{1+\left|\epsilon_{L}\right|^{2}}=\frac{a_{\epsilon}+a_{\Delta}}{1+a_{\epsilon} a_{\Delta}} \\
\eta_{\Delta} & =\frac{2 \Delta_{M}}{1+\Delta_{M}^{2}}=\frac{a_{\Delta}+i a_{\Delta}^{\prime} \sqrt{1-a_{\Delta}^{2}-a_{\Delta}^{\prime 2}}}{1-a_{\Delta}^{\prime 2}}
\end{aligned}
$$

with

$$
\begin{aligned}
a_{\epsilon} & =\frac{1-|q / p|^{2}}{1+|q / p|^{2}}=\frac{2 R e \epsilon_{M}}{1+\left|\epsilon_{M}\right|^{2}} \\
a_{\Delta} & =\frac{2 \operatorname{Re} \Delta_{M}}{1+\left|\Delta_{M}\right|^{2}}, \quad a_{\Delta}^{\prime}=\frac{2 \operatorname{Im} \Delta_{M}}{1+\left|\Delta_{M}\right|^{2}}
\end{aligned}
$$

The definitions of $\hat{a}_{\epsilon^{\prime}}, \hat{a}_{\epsilon_{S}+\epsilon^{\prime}}$ and $\hat{a}_{\epsilon_{S} \epsilon^{\prime}}$ are given in the appendix. The reader should note that quantities without a hat contain either only $\mathrm{CP}$ or only CPT nonconserving effects, and with a hat contain both CP- and CPT-nonconserving effects.

As $a_{\epsilon}, \hat{a}_{\epsilon^{\prime}}, \hat{a}_{\epsilon+\epsilon^{\prime}}$ and $\hat{a}_{\epsilon \epsilon^{\prime}}$ (for their definitions see appendix) are all rephase-invariant, so are also $\hat{a}_{\epsilon_{S}+\epsilon^{\prime}}$ and $\hat{a}_{\epsilon_{S} \epsilon^{\prime}}$. Note that only three of them are independent since $\left(1-a_{\epsilon}^{2}\right)\left(1-\hat{a}_{\epsilon^{\prime}}^{2}\right)=$ $\hat{a}_{\epsilon+\epsilon^{\prime}}^{2}+\left(1+\hat{a}_{\epsilon \epsilon^{\prime}}\right)^{2}$. Another rephase-invariant direct CP and CPT noninvariant observable is defined as

$$
\hat{a}_{\epsilon^{\prime \prime}}=\frac{1-|\bar{g} / g|^{2}}{1+|\bar{g} / g|^{2}}=\frac{2 \operatorname{Re} \varepsilon_{M}^{\prime \prime}}{1+\left|\varepsilon_{M}^{\prime \prime}\right|^{2}}=\frac{a_{\epsilon^{\prime \prime}}+a_{\varepsilon \Delta}+a_{\Delta \Delta}^{\prime}}{1+a_{\varepsilon \Delta}^{\prime}+a_{\Delta \Delta}}
$$

where the definitions for $a_{\epsilon^{\prime \prime}}, a_{\varepsilon \Delta}, a_{\Delta \Delta}^{\prime}, a_{\varepsilon \Delta}^{\prime}$ and $a_{\Delta \Delta}$ are presented in the appendix. Analogously, one has

$$
\hat{\eta}_{\bar{f}} \equiv \frac{q_{S}}{q_{L}} \frac{<\bar{f}\left|H_{e f f}\right| M_{2}>}{<\bar{f}\left|H_{e f f}\right| M_{1}>}=\frac{1}{1-\eta_{\Delta}}\left[\eta_{\Delta}+\frac{a_{\epsilon_{S}}+\hat{a}_{\bar{\varepsilon}^{\prime}}+i \hat{a}_{\epsilon_{S}+\bar{\varepsilon}^{\prime}}}{2+a_{\epsilon_{S}} \hat{a}_{\bar{\varepsilon}^{\prime}}+\hat{a}_{\epsilon_{S} \bar{\varepsilon}^{\prime}}}\right]
$$

and

$$
\hat{a}_{\bar{\epsilon}^{\prime \prime}}=\frac{1-|\bar{h} / h|^{2}}{1+|\bar{h} / h|^{2}}=\frac{2 R e \bar{\varepsilon}_{M}^{\prime \prime}}{1+\left|\bar{\varepsilon}_{M}^{\prime \prime}\right|^{2}}=\frac{a_{\bar{\epsilon}^{\prime \prime}}+a_{\bar{\varepsilon} \bar{\Delta}}+a_{\bar{\Delta} \bar{\Delta}}^{\prime}}{1+a_{\bar{\varepsilon} \bar{\Delta}}^{\prime}+a_{\bar{\Delta} \bar{\Delta}}}
$$

\footnotetext{
${ }^{1}$ The algebra is described in ref. 11 .
} 
with $\bar{\Delta}_{i}=D_{i} / C_{i}$.

One of the interesting cases occurs when the final states are CP eigenstates, i.e., $f^{C P}=f$, and in this case $h=\bar{g}$ ( or $C=A$ and $D=B$ ). As a consequence, we find

$$
\begin{aligned}
& \hat{a}_{\epsilon^{\prime}}=\hat{a}_{\epsilon^{\prime \prime}}, \quad a_{\epsilon^{\prime}}=a_{\epsilon^{\prime \prime}} \\
& \hat{a}_{\epsilon+\epsilon^{\prime}}=\frac{1}{1+a_{\varepsilon \Delta}^{\prime}+a_{\Delta \Delta}}\left[a_{\epsilon+\epsilon^{\prime}}+a_{\epsilon+\epsilon_{\Delta}^{\prime}}+a_{\epsilon+\epsilon_{\Delta \Delta}^{\prime}}\right]
\end{aligned}
$$

where the explicit definitions for $a_{\epsilon+\epsilon^{\prime}}, a_{\epsilon+\epsilon_{\Delta}^{\prime}}$ and $a_{\epsilon+\epsilon_{\Delta \Delta}^{\prime}}$ are again given in the appendix.

To see explicitly how many rephase invariant CPT and CP observables may be separately measured from experiments, let us consider the case for which the final states are CP eigenstates and suppose that the violations are small so that one could only keep the linear terms of the rephase invariant CPT- and CP-violating observables. With this consideration, the observable $\hat{\eta}_{f}$ is simplified

$$
\hat{\eta}_{f} \simeq \frac{1}{2}\left[a_{\epsilon}+a_{\epsilon^{\prime}}+a_{\Delta}+a_{\epsilon \Delta}+a_{\Delta \Delta}^{\prime}+i\left(a_{\epsilon+\epsilon^{\prime}}+a_{\Delta}^{\prime}+a_{\epsilon+\epsilon_{\Delta \Delta}^{\prime}}+a_{\epsilon+\epsilon_{\Delta}^{\prime}}\right)\right]
$$

where the definitions for all the rephase invariant quantities are given in the appendix. Those with index $\Delta$ are the CPT-violating observables, the others are CP-violating ones which have been discussed in ref. 11.

The formalism so far involves many equations which include CP and CPT violation effects either separately or mixed together. It has several advantages in comparison with other articles [12, 10]:

1. The formalism is more general than the ones reported in the literature and can be applied not only to the K-meson decays but also all other heavier meson decays.

2. All observables are manifestly rephasing invariant and well defined by directly relating to the hadronic mixing matrix elements and decay amplitudes of mesons.

3. All possible independent observables are classified, which enables one to separately measure different types of CPT- and CP-violating observables and to extract purely CPT or CP violation effects.

4. The formalism is more elegantly designed for extracting various rephase invariant CPT- and CP- violating observables from time-dependent measurements of meson decays, which will be discussed in detail in the next section.

We have thus defined all possible rephase-invariant CP and CPT noninvariant observables in terms of eight parameters related to $\mathrm{CP}$ and $\mathrm{CPT}$ breaking quantities arising either from mixing or phases of amplitude. The eight parameters are classified as follows: $\epsilon_{M}$ is an indirect CP-violating parameter and $\Delta_{M}$ the indirect CPT-violating parameters; the parameters $\varepsilon_{M}^{\prime \prime}$ and $\bar{\varepsilon}_{M}^{\prime \prime}$ will be decomposed into four parameters, $\epsilon_{M}^{\prime \prime}, \bar{\epsilon}_{M}^{\prime \prime}, \Delta_{i}$ and 
$\bar{\Delta}_{i}$, where $\epsilon_{M}^{\prime \prime}$ and $\bar{\epsilon}_{M}^{\prime \prime}$ define direct CP-violating paramters, $\Delta_{i}$ and $\bar{\Delta}_{i}$ describe direct CPT-violating parameters. $\varepsilon_{M}^{\prime}$ and $\bar{\varepsilon}_{M}^{\prime}$ contain the ratio of the two decay amplitudes and can be associated with direct $\mathrm{CP}$ and $\mathrm{CPT}$ violation, as well as the interference between indirect and direct $\mathrm{CP}$ and $\mathrm{CPT}$ violations. All the $\mathrm{CP}$ and $\mathrm{CPT}$ violations can be well defined and in general classified into the following types:

1. purely indirect $\mathrm{CP}$ and $\mathrm{CPT}$ violations which are given by the rephase-invariant CP-violating observable $a_{\epsilon}$ and CPT-violating observables $a_{\Delta}$ and $a_{\Delta}^{\prime}$.

2. purely direct $\mathrm{CP}$ and $\mathrm{CPT}$ violations which are characterized by the rephase-invariant CP-violating observables $a_{\epsilon^{\prime \prime}}$ and $a_{\bar{\epsilon}^{\prime \prime}}$ and CPT-violating observables $a_{\varepsilon \Delta}, a_{\varepsilon \Delta}^{\prime}, a_{\Delta \Delta}$, $a_{\Delta \Delta}^{\prime}, a_{\bar{\varepsilon} \bar{\Delta}}, a_{\bar{\varepsilon} \bar{\Delta}}^{\prime}, a_{\bar{\Delta} \bar{\Delta}}$ and $a_{\bar{\Delta} \bar{\Delta}}^{\prime}$.

3. Mixed-induced $\mathrm{CP}$ and $\mathrm{CPT}$ violations which are described by CP-violating observables $a_{\epsilon+\epsilon^{\prime}}$ and $a_{\epsilon+\bar{\epsilon}^{\prime}}$ and CPT-violating observables $a_{\epsilon+\epsilon_{\Delta}^{\prime}}, a_{\epsilon+\epsilon_{\Delta \Delta}^{\prime}}, a_{\epsilon+\epsilon_{\Delta}^{\prime}}$ and $a_{\epsilon+\bar{\epsilon}_{\Delta \Delta}^{\prime}} \cdot$

For the case that the final states are CP eigenstates, one has $\hat{a}_{\epsilon^{\prime}}=\hat{a}_{\epsilon^{\prime \prime}}=\hat{a}_{\bar{\epsilon}^{\prime}}=\hat{a}_{\bar{\epsilon}^{\prime \prime}}$. Thus, in this case $\hat{a}_{\epsilon^{\prime}}$ and $\hat{a}_{\bar{\epsilon}^{\prime}}$ also indicate purely direct $\mathrm{CP}$ and $\mathrm{CPT}$ violations. When the final states are not CP eigenstates, $\hat{a}_{\epsilon^{\prime}}$ and $\hat{a}_{\bar{\epsilon}^{\prime}}$ do not, in general, provide a clear signal of direct $\mathrm{CP}$ violation although they contain direct $\mathrm{CP}$ and $\mathrm{CPT}$ violations. Their deviation from the values $\hat{a}_{\epsilon^{\prime}}= \pm 1,0$ and $\hat{a}_{\bar{\epsilon}^{\prime}}=\mp 1,0$ can arise from different CKM angles, final state interactions, or different hadronic form factors, but not necessarily from $\mathrm{CP}$ and $\mathrm{CPT}$ violations.

\section{Extraction of CP- and CPT-violating Observables}

In order to measure the rephase-invariant observables defined above, we consider the proper time evolution [13, [14] of the neutral mesons

$$
\left|M^{0}(t)>=\sum_{i=1}^{2} \xi_{i} e^{-i\left(m_{i}-i \Gamma_{i} / 2\right) t}\right| M_{i}>; \quad\left|\bar{M}^{0}(t)>=\sum_{i=1}^{2} \bar{\xi}_{i} e^{-i\left(m_{i}-i \Gamma_{i} / 2\right) t}\right| M_{i}>
$$

with $\xi_{1}=q_{L} /\left(q_{S} p_{L}+q_{L} p_{S}\right)$ and $\xi_{2}=q_{S} /\left(q_{S} p_{L}+q_{L} p_{S}\right)$ for a pure $M^{0}$ state at $t=0$ as well as $\bar{\xi}_{1}=p_{L} /\left(q_{S} p_{L}+q_{L} p_{S}\right)$ and $\bar{\xi}_{2}=-p_{S} /\left(q_{S} p_{L}+q_{L} p_{S}\right)$ for a pure $\bar{M}^{0}$ state at $t=0$. Thus the decay amplitudes of $M^{0}$ and $\bar{M}^{0}$ at the time $t$ will be given by

$$
\begin{aligned}
& \mathcal{A}(t)=<f \mid M^{0}(t)>=\frac{<f \mid M_{1}>\frac{1-\eta_{\Delta}}{p_{S}}\left(e^{-i H_{1} t}+\hat{\eta}_{f} e^{-i H_{2} t}\right)}{2} \\
& \overline{\mathcal{A}}(t)=<\bar{f} \mid \bar{M}^{0}(t)>=\frac{<\bar{f} \mid M_{1}>}{q_{S}} \frac{1-\eta_{\Delta}}{2}\left(\frac{1+\eta_{\Delta}}{1-\eta_{\Delta}} e^{-i H_{1} t}-\hat{\eta}_{\bar{f}} e^{-i H_{2} t}\right)
\end{aligned}
$$


It follows now that the time-dependent decay rates are

$$
\begin{aligned}
& \Gamma\left(M^{0}(t) \rightarrow f\right) \propto|\mathcal{A}(t)|^{2}=\left(|g|^{2}+|h|^{2}\right) \frac{2+a_{\epsilon_{S}} \hat{a}_{\epsilon^{\prime}}+\hat{a}_{\epsilon_{S} \epsilon^{\prime}}}{1+a_{\epsilon_{S}}} e^{-\Gamma t} \\
& \cdot\left\{\left[\frac{1+a_{\epsilon_{S}} \hat{a}_{\epsilon^{\prime}}+\left(a_{\epsilon_{S}}+\hat{a}_{\epsilon^{\prime}}\right) R e \eta_{\Delta}+\hat{a}_{\epsilon_{S}+\epsilon^{\prime}} I m \eta_{\Delta}}{2+a_{\epsilon_{S}} \hat{a}_{\epsilon^{\prime}}+\hat{a}_{\epsilon_{S} \epsilon^{\prime}}}-\operatorname{Re} \eta_{\Delta}+\left|\eta_{\Delta}\right|^{2}\right] \cosh (\Delta \Gamma t)\right. \\
& +\left[\frac{1+a_{\epsilon_{S} \hat{\epsilon}^{\prime}}-\left(a_{\epsilon_{S}}+\hat{a}_{\epsilon^{\prime}}\right) R e \eta_{\Delta}-\hat{a}_{\epsilon_{S}+\epsilon^{\prime}} I m \eta_{\Delta}}{2+a_{\epsilon_{S}} \hat{a}_{\epsilon^{\prime}}+\hat{a}_{\epsilon_{S} \epsilon^{\prime}}}-\operatorname{Re} \eta_{\Delta}\right] \sinh (\Delta \Gamma t) \\
& +\left[\frac{\left(a_{\epsilon_{S}}+\hat{a}_{\epsilon^{\prime}}\right)\left(1-R e \eta_{\Delta}\right)-\hat{a}_{\epsilon_{S}+\epsilon^{\prime}} I m \eta_{\Delta}}{2+a_{\epsilon_{S}} \hat{a}_{\epsilon^{\prime}}+\hat{a}_{\epsilon_{S} \epsilon^{\prime}}}+\operatorname{Re} \eta_{\Delta}-\left|\eta_{\Delta}\right|^{2}\right] \cos (\Delta m t) \\
& \left.+\left[\frac{\hat{a}_{\epsilon_{S}+\epsilon^{\prime}}\left(1-R e \eta_{\Delta}\right)+\left(a_{\epsilon_{S}}+\hat{a}_{\epsilon^{\prime}}\right) I m \eta_{\Delta}}{2+a_{\epsilon_{S}} \hat{a}_{\epsilon^{\prime}}+\hat{a}_{\epsilon_{S} \epsilon^{\prime}}}+I m \eta_{\Delta}\right] \sin (\Delta m t)\right\}
\end{aligned}
$$

and

$$
\begin{aligned}
& \Gamma\left(\bar{M}^{0}(t) \rightarrow \bar{f}\right) \propto|\overline{\mathcal{A}}(t)|^{2}=\left(|\bar{g}|^{2}+|\bar{h}|^{2}\right) \frac{2+a_{\epsilon_{S}} \hat{a}_{\bar{\epsilon}^{\prime}}+\hat{a}_{\epsilon_{S} \bar{\epsilon}^{\prime}}}{1+a_{\epsilon_{S}}} e^{-\Gamma t} \\
& \cdot\left\{\left[\frac{1+a_{\epsilon_{S}} \hat{a}_{\bar{\epsilon}^{\prime}}+\left(a_{\epsilon_{S}}+\hat{a}_{\bar{\epsilon}^{\prime}}\right) \operatorname{Re} \eta_{\Delta}+\hat{a}_{\epsilon_{S}+\bar{\epsilon}^{\prime}} I m \eta_{\Delta}}{2+a_{\epsilon_{S}} \hat{a}_{\bar{a}^{\prime}}+\hat{a}_{\epsilon_{S} \bar{\epsilon}^{\prime}}}-\operatorname{Re} \eta_{\Delta}-\left|\eta_{\Delta}\right|^{2}\right] \cosh (\Delta \Gamma t)\right. \\
& +\left[\frac{1+a_{\epsilon_{S} \hat{\epsilon}^{\prime}}-\left(a_{\epsilon_{S}}+\hat{a}_{\bar{\epsilon}^{\prime}}\right) \operatorname{Re} \eta_{\Delta}-\hat{a}_{\epsilon_{S}+\bar{\epsilon}^{\prime}} I m \eta_{\Delta}}{2+a_{\epsilon_{S}} \hat{a}_{\bar{\epsilon}^{\prime}}+\hat{a}_{\epsilon_{S} \bar{\epsilon}^{\prime}}}-\operatorname{Re} \eta_{\Delta}\right] \sinh (\Delta \Gamma t) \\
& -\left[\frac{\left(a_{\epsilon_{S}}+\hat{a}_{\bar{\epsilon}^{\prime}}\right)\left(1+R e \eta_{\Delta}\right)+\hat{a}_{\epsilon_{S}+\bar{\epsilon}^{\prime}} I m \eta_{\Delta}}{2+a_{\epsilon_{S}} \hat{a}_{\bar{\epsilon}^{\prime}}+\hat{a}_{\epsilon_{S} \bar{\epsilon}^{\prime}}}+R e \eta_{\Delta}+\left|\eta_{\Delta}\right|^{2}\right] \cos (\Delta m t) \\
& \left.-\left[\frac{\hat{a}_{\epsilon_{S}+\bar{\epsilon}^{\prime}}\left(1+\operatorname{Re} \eta_{\Delta}\right)-\left(a_{\epsilon_{S}}+\hat{a}_{\bar{\epsilon}^{\prime}}\right) I m \eta_{\Delta}}{2+a_{\epsilon_{S}} \hat{a}_{\bar{\epsilon}^{\prime}}+\hat{a}_{\epsilon_{S} \bar{\epsilon}^{\prime}}}-I m \eta_{\Delta}\right] \sin (\Delta m t)\right\}
\end{aligned}
$$

where $\Delta \Gamma=\Gamma_{2}-\Gamma_{1}$ and $\Delta m=m_{2}-m_{1}$. Here we have omitted the integrals from the phase space. Similarly, one can easily write down the decay rates $\Gamma\left(M^{0}(t) \rightarrow \bar{f}\right)$ and $\Gamma\left(\bar{M}^{0}(t) \rightarrow f\right)$, and then the time-dependent CP and CPT asymmetries are defined by the difference between two decay rates. In addition, in studies of the time dependence one can isolate each of four-terms. One can introduce several asymmetries from the decay rates $\Gamma\left(M^{0}(t) \rightarrow f\right), \Gamma\left(\bar{M}^{0}(t) \rightarrow \bar{f}\right), \Gamma\left(M^{0}(t) \rightarrow \bar{f}\right)$ and $\Gamma\left(\bar{M}^{0}(t) \rightarrow f\right)$. Obviously, the time dependences contains a lot of information. Therefore studies of time evolution can eliminate the various components (hamonics) in $\cos (\Delta m t), \sin (\Delta m t), \cosh (\Delta \Gamma t)$ and $\sinh (\Delta \Gamma t)$. We now proceed to apply the above general analysis to specific processes. As in the ref.[1], we may classify the processes into four scenarios:

i) $M^{0} \rightarrow f\left(M^{0} \nrightarrow \rightarrow \bar{f}\right), \bar{M}^{0} \rightarrow \bar{f}\left(\bar{M}^{0} \nrightarrow \rightarrow f\right)$, this is the case when $f$ and $\bar{f}$ are not a common final state of $M^{0}$ and $\bar{M}^{0}$. Examples are: $M^{0} \rightarrow M^{-} \bar{l} \nu, \bar{M}^{0} \rightarrow M^{\prime+} l \bar{\nu}$; $B^{0} \rightarrow D^{-} D_{s}^{+}, D^{-} K^{+}, \pi^{-} D_{s}^{+}, \pi^{-} K^{+}, \bar{B}^{0} \rightarrow D^{+} D_{s}^{-}, D^{+} K^{-}, \pi^{+} D_{s}^{-}, \pi^{+} K^{-} ; B_{s}^{0} \rightarrow D_{s}^{-} \pi^{+}$, $D_{s}^{-} D^{+}, K^{-} \pi^{+}, K^{-} D^{+}, \bar{B}_{s}^{0} \rightarrow D_{s}^{+} \pi^{-}, D_{s}^{+} D^{-}, K^{+} \pi^{-}, K^{+} D^{-}$. This scenario also applies to charged meson decays.

ii) $M^{0} \rightarrow\left(f=\bar{f}, f^{C P}=f\right) \leftarrow \bar{M}^{0}$, this is the decay to a common final state which is CP eigenstate. Such as $B^{0}\left(\bar{B}^{0}\right), D^{0}\left(\bar{D}^{0}\right), K^{0}\left(\bar{K}^{0}\right) \rightarrow \pi^{+} \pi^{-}, \pi^{0} \pi^{0}, \cdots$. For the final 
states such as $\pi^{-} \rho^{+}$and $\pi^{+} \rho^{-}$, although each of them is not a CP eigenstate of $B^{0}\left(\bar{B}^{0}\right)$ or $D^{0}\left(\bar{D}^{0}\right)$, one can always decompose them into CP eigenstates as $(\pi \rho)_{ \pm}=\left(\pi^{-} \rho^{+} \pm \pi^{+} \rho^{-}\right)$ with $C P(\pi \rho)_{ \pm}= \pm(\pi \rho)_{ \pm}$. This reconstruction is meaningful since $\pi^{-} \rho^{+}$and $\pi^{+} \rho^{-}$have the same weak phase as they contain the same quark content.

iii) $M^{0} \rightarrow\left(f, f \not \rightarrow f^{C P}\right) \leftarrow \bar{M}^{0}$, i.e., the final states are common final states but are not charge conjugate states. For example, $B^{0}\left(\bar{B}^{0}\right) \rightarrow K_{S} J / \psi, B_{s}^{0}\left(\bar{B}_{s}^{0}\right) \rightarrow K_{S} \phi$ and $D^{0}\left(\bar{D}^{0}\right) \rightarrow K_{S} \pi^{0}, K_{S} \rho^{0}$.

iv) $M^{0} \rightarrow\left(f \& \bar{f}, f^{C P} \neq f\right) \leftarrow \bar{M}^{0}$, i.e., both $f$ and $\bar{f}$ are the common final states of $M^{0}$ and $\bar{M}^{0}$, but they are not CP eigenstates. This is the most general case. For example, $B^{0}\left(\bar{B}^{0}\right) \rightarrow D^{-} \pi^{+}, \pi^{-} D^{+} ; D^{-} \rho^{+}, \rho^{-} D^{+} ; B_{s}^{0}\left(\bar{B}_{s}^{0}\right) \rightarrow D_{s}^{-} K^{+}, K^{-} D_{s}^{+} ; D^{0}\left(\bar{D}^{0}\right) \rightarrow K^{-} \pi^{+}$, $K^{+} \pi^{-}$.

In this paper, we will only elaborate on the first two scenarios. In scenario i), the amplitudes $h$ and $\bar{h}$ are zero, thus $\hat{a}_{\epsilon^{\prime}}=-\hat{a}_{\bar{\epsilon}^{\prime}}=1, \hat{a}_{\epsilon+\epsilon^{\prime}}=0=\hat{a}_{\epsilon+\bar{\epsilon}^{\prime}}$ and $\hat{a}_{\epsilon \epsilon^{\prime}}=-1=\hat{a}_{\epsilon \bar{\epsilon}^{\prime}}$. For this case, the time-dependent rates of eqs.(29) and (30) will become very simple,

$$
\begin{aligned}
\Gamma\left(M^{0}(t) \rightarrow f\right) & \propto|\mathcal{A}(t)|^{2}=|g|^{2} e^{-\Gamma t} \cdot\left\{\left(1+\left|\eta_{\Delta}\right|^{2}\right) \cosh \Delta \Gamma t\right. \\
& \left.-2 R e \eta_{\Delta} \sinh \Delta \Gamma t+\left(1-\left|\eta_{\Delta}\right|^{2}\right) \cos \Delta m t+I m \eta_{\Delta} \sin \Delta m t\right\} \\
\Gamma\left(\bar{M}^{0}(t) \rightarrow \bar{f}\right) & \propto|\overline{\mathcal{A}}(t)|^{2}=|\bar{g}|^{2} e^{-\Gamma t} \cdot\left\{\left(1+\left|\eta_{\Delta}\right|^{2}\right) \cosh \Delta \Gamma t\right. \\
& \left.+2 R e \eta_{\Delta} \sinh \Delta \Gamma t+\left(1-\left|\eta_{\Delta}\right|^{2}\right) \cos \Delta m t-I m \eta_{\Delta} \sin \Delta m t\right\}
\end{aligned}
$$

It is not difficult to show that the other two time-dependent decay rates which are not allowed at $t=0$, can happen at a later $t$, because the $M^{0}$ develops an $\bar{M}^{0}$ component through mixing. They can be simply expressed as

$$
\begin{aligned}
\Gamma\left(M^{0}(t) \rightarrow \bar{f}\right) \propto & \frac{g^{2}+|\bar{g}|^{2}}{2}\left(1-\hat{a}_{\epsilon^{\prime \prime}}\right)\left(\frac{1-a_{\epsilon_{S}}}{1+a_{\epsilon_{S}}}\right) \frac{\left(1-a_{\Delta}\right)^{2}}{1-a_{\Delta}^{\prime 2}} \\
& \cdot e^{-\Gamma t}(\cosh \Delta \Gamma t-\cos \Delta m t) \\
\Gamma\left(\bar{M}^{0}(t) \rightarrow f\right) \propto & \frac{g^{2}+|\bar{g}|^{2}}{2}\left(1+\hat{a}_{\epsilon^{\prime \prime}}\right)\left(\frac{1+a_{\epsilon_{L}}}{1-a_{\epsilon_{L}}}\right) \frac{\left(1-a_{\Delta}\right)^{2}}{1-a_{\Delta}^{\prime 2}} \\
& \cdot e^{-\Gamma t}(\cosh \Delta \Gamma t-\cos \Delta m t)
\end{aligned}
$$

With these four decay rates, we can define three asymmetries which have the following simple forms when neglecting the quadratic and high order terms of the $\mathrm{CP}$ and $\mathrm{CPT}$ violating parameters (i.e., $a_{\Delta}^{2}, a_{\Delta}^{\prime}, a_{\epsilon} a_{\Delta}^{2}$ )

$$
\begin{aligned}
A_{C P+C P T}(t) & =\frac{\Gamma\left(M^{0}(t) \rightarrow f\right)-\Gamma\left(\bar{M}^{0}(t) \rightarrow \bar{f}\right)}{\Gamma\left(M^{0}(t) \rightarrow f\right)+\Gamma\left(\bar{M}^{0}(t) \rightarrow \bar{f}\right)} \\
& \simeq a_{\epsilon^{\prime \prime}}+a_{\varepsilon \Delta}+\frac{-a_{\Delta} \sinh \Delta \Gamma t+a_{\Delta}^{\prime} \sin \Delta m t}{\cosh \Delta \Gamma t+\cos \Delta m t} \\
A_{C P+C P T}^{\prime}(t) & =\frac{\Gamma\left(\bar{M}^{0}(t) \rightarrow f\right)-\Gamma\left(M^{0}(t) \rightarrow \bar{f}\right)}{\Gamma\left(\bar{M}^{0}(t) \rightarrow f\right)+\Gamma\left(M^{0}(t) \rightarrow \bar{f}\right)}
\end{aligned}
$$




$$
\begin{aligned}
& \simeq a_{\epsilon^{\prime \prime}}+a_{\varepsilon \Delta}+2 a_{\epsilon} \\
A_{C P+C P T}^{\prime \prime}(t) & =\frac{\Gamma\left(M^{0}(t) \rightarrow f\right)-\Gamma\left(\bar{M}^{0}(t) \rightarrow f\right)}{\Gamma\left(M^{0}(t) \rightarrow f\right)+\Gamma\left(\bar{M}^{0}(t) \rightarrow f\right)} \\
& \simeq \frac{\cos \Delta m t-a_{\epsilon} \cosh \Delta \Gamma t-a_{\Delta} \sinh \Delta \Gamma t+a_{\Delta}^{\prime} \sin \Delta m t}{\cosh \Delta \Gamma t-a_{\epsilon} \cos \Delta m t-a_{\Delta} \sinh \Delta \Gamma t+a_{\Delta}^{\prime} \sin \Delta m t}
\end{aligned}
$$

Their exact expressions can be found in the appendix. From the time-dependent measurements of the above asymmetries, one shall be able to extract all observables: $\Delta m$, $\Delta \Gamma, a_{\epsilon}, a_{\Delta}, a_{\Delta}^{\prime}$ and $\hat{a}_{\epsilon^{\prime \prime}}$

From the above asymmetries, we easily arrive at the following important observations:

1. As long as the experimental measurements show that the asymmetry $A_{C P+C P T}(t)$ is not a constant and depends on time, it provides a clean signature of indirect CPT violation from mixings.

2. For the semileptonic decays $M^{0} \rightarrow M^{\prime}-l \nu$ and also for the decay modes in which the final state interactions are absent, one has $a_{\epsilon^{\prime \prime}}=0, a_{\Delta \Delta}^{\prime}=0, a_{\varepsilon \Delta}^{\prime}=0$ and $\hat{a}_{\epsilon^{\prime \prime}}=a_{\varepsilon \Delta} /\left(1+a_{\Delta \Delta}\right)$, thus nonzero $\hat{a}_{\epsilon^{\prime \prime}}$ will represent direct CPT violation from amplitudes. For this case, we come to a strong conclusion that once the asymmetry $A_{C P+C P T}(t)$ is not zero, then CPT must be violated.

3. By combining measurement of the above asymmetries from semileptonic and nonleptonic decays, it allows one, in principle, to separately measure the indirect CPviolating observable $a_{\epsilon}$ and the direct CP-violating observable $a_{\epsilon^{\prime \prime}}$ as well as the indirect CPT-violating observables $a_{\Delta}$ and $a_{\Delta}^{\prime}$, and the direct CPT-violating observable $a_{\epsilon \Delta}$.

We now discuss scenario ii) in which $\bar{h}=g$ and $h=\bar{g}$, thus $a_{\epsilon^{\prime}}=a_{\epsilon^{\prime \prime}}=a_{\bar{\epsilon}^{\prime}}=a_{\bar{\epsilon}^{\prime \prime}}$ and $a_{\epsilon+\epsilon^{\prime}}=a_{\epsilon+\bar{\epsilon}^{\prime}}$. When neglecting the quadratic and high order terms and using the relations and definitions for the rephase-invariant observables, the time-dependent asymmetry is simply given by

$$
A_{C P+C P T}(t) \simeq-\left(a_{\epsilon}+a_{\Delta}\right)+e^{-\Delta \Gamma t}\left[\left(a_{\epsilon}+a_{\Delta}+\hat{a}_{\epsilon^{\prime}}\right) \cos (\Delta m t)+\left(a_{\Delta}^{\prime}+\hat{a}_{\epsilon+\epsilon^{\prime}}\right) \sin (\Delta m t)\right]
$$

(The exact expression is given in the appendix.)

From the above time-dependent evolution $A_{C P+C P T}(t)$ one is able to extract three physical quantities: one of them is the direct $\mathrm{CP}$ and CPT noninvariant observable $\hat{a}_{\epsilon^{\prime}}$ and the other two are the combinations of CP and CPT noninvariant observables $\left(a_{\epsilon}+a_{\Delta}\right)$ and $\left(a_{\Delta}^{\prime}+\hat{a}_{\epsilon+\epsilon^{\prime}}\right)$. Combining these measurements with scenario (i), in which the indirect CP and CPT noninvariant observables $a_{\epsilon}, a_{\Delta}$ and $a_{\Delta}^{\prime}$ are expected to be determined, one will be able to extract the mixed-induced CP and CPT noninvariant observable $\hat{a}_{\epsilon+\epsilon^{\prime}}$. Thus, studies of scenarios (i) and (ii) allow us to separate the three types of CP and CPT violations. 


\section{$6 \mathrm{CP}$ and CPT Violation in K-meson System}

The formalism and analyses presented above are general and can be used for all neutral meson systems. As a specific application, we are going to consider the K-meson system. From semileptonic decays of $K^{0} \rightarrow \pi^{-}+l^{+}+\nu_{l}$ and $\bar{K}^{0} \rightarrow \pi^{+}+l^{-}+\overline{\nu_{l}}$, from eqs.(33) and (34), the time-dependent measurements of the asymmetries lead to

$$
\begin{aligned}
A_{C P+C P T}^{K_{l 3}}(t) & =\frac{\Gamma\left(K^{0}(t) \rightarrow \pi^{-} l^{+} \nu_{l}\right)-\Gamma\left(\bar{K}^{0}(t) \rightarrow \pi^{+} l^{-} \overline{\nu_{l}}\right)}{\Gamma\left(K^{0}(t) \rightarrow \pi^{-} l^{+} \nu_{l}\right)+\Gamma\left(\bar{K}^{0}(t) \rightarrow \pi^{+} l^{-} \bar{\nu}_{l}\right)} \\
& \simeq a_{\varepsilon \Delta}+\frac{-a_{\Delta} \sinh \Delta \Gamma t+a_{\Delta}^{\prime} \sin \Delta m_{K} t}{\cosh \Delta \Gamma t+\cos \Delta m_{K} t}, \\
A_{C P+C P T}^{\prime K_{l 3}}(t) & =\frac{\Gamma\left(\bar{K}^{0}(t) \rightarrow \pi^{-} l^{+} \nu_{l}\right)-\Gamma\left(K^{0}(t) \rightarrow \pi^{+} l^{-} \bar{\nu}_{l}\right)}{\Gamma\left(\bar{K}^{0}(t) \rightarrow \pi^{-} l^{+} \nu_{l}\right)+\Gamma\left(K^{0}(t) \rightarrow \pi^{+} l^{-} \bar{\nu}_{l}\right)} \\
& \simeq a_{\varepsilon \Delta}+2 a_{\epsilon}
\end{aligned}
$$

where the direct CP-violating parameter $a_{\epsilon^{\prime \prime}}$ is expected to be small as the final state interactions are electromagnetic. It is then clear that non-zero asymmetry $A_{C P+C P T}^{K_{l 3}}(t)$ is a clean signature of CPT violation. Its time evolution allows us to extract direct CPT-violating observable $a_{\varepsilon \Delta}$ and indirect CPT-violating observables $a_{\Delta}$ and $a_{\Delta}^{\prime}$. The combination of the two asymmetries $A_{C P+C P T}^{\prime} K_{l 3}(t)$ and $A_{C P+C P T}^{K_{l 3}}(t)$ further helps us to extract indirect CP-violating observable $a_{\epsilon}$.

In the nonleptonic decays with final states being CP eigenstates, the asymmetry $A_{C P+C P T}(t)$ is given in terms of the observables $\hat{a}_{\epsilon^{\prime}}$ and $\hat{a}_{\epsilon+\epsilon^{\prime}}$ which concern both CP and CPT violations. In general, it is hard to clearly separate $\mathrm{CP}$ violation from $\mathrm{CPT}$ violation in the decay amplitudes, but it would be of interest to look for possibilities of establishing CPT violation arising from the decay amplitudes. For the K-meson system, there are two unique decay modes $K^{0}\left(\bar{K}^{0}\right) \rightarrow \pi^{+} \pi^{-}$and $\pi^{0} \pi^{0}$ which are related via isospin symmetry. Their time-dependent asymmetries are given by

$$
\begin{aligned}
& A_{C P+C P T}^{\left(\pi^{+} \pi^{-}\right)}(t) \simeq-\left(a_{\epsilon}+a_{\Delta}\right) \\
& +e^{-\Delta \Gamma t}\left[\left(a_{\epsilon}+a_{\Delta}+\hat{a}_{\epsilon^{\prime}}^{(+-)}\right) \cos \left(\Delta m_{K} t\right)+\left(a_{\Delta}^{\prime}+\hat{a}_{\epsilon+\epsilon^{\prime}}^{(+-)}\right) \sin \left(\Delta m_{K} t\right)\right], \\
& A_{C P+C P T}^{\left(\pi^{0} \pi^{0}\right)}(t) \simeq-\left(a_{\epsilon}+a_{\Delta}\right) \\
& +e^{-\Delta \Gamma t}\left[\left(a_{\epsilon}+a_{\Delta}+\hat{a}_{\epsilon^{\prime}}^{(00)}\right) \cos \left(\Delta m_{K} t\right)+\left(a_{\Delta}^{\prime}+\hat{a}_{\epsilon+\epsilon^{\prime}}^{(00)}\right) \sin \left(\Delta m_{K} t\right)\right] .
\end{aligned}
$$

It is seen that since the indirect CP-violating observable $a_{\epsilon}$ and indirect CPT-violating observables $a_{\Delta}$ and $a_{\Delta}^{\prime}$ can be extracted from asymmetries in the semileptonic decays, we then can extract the direct CP- and CPT-violating observables $\hat{a}_{\epsilon^{\prime}}^{(+-)}$and $\hat{a}_{\epsilon^{\prime}}^{(00)}$ as well as mixed-induced CP- and CPT-violating observables $\hat{a}_{\epsilon+\epsilon^{\prime}}^{(+-)}$and $\hat{a}_{\epsilon+\epsilon^{\prime}}^{(00)}$. We now discuss how to extract pure $\mathrm{CPT}$ or $\mathrm{CP}$ violation effects by using isospin symmetry.

When neglecting high order terms, we have

$$
\hat{a}_{\epsilon^{\prime}} \simeq a_{\epsilon^{\prime}}+a_{\Delta \Delta}^{\prime}+a_{\varepsilon \Delta}, \quad \hat{a}_{\epsilon+\epsilon^{\prime}} \simeq a_{\epsilon+\epsilon^{\prime}}+a_{\epsilon+\epsilon_{\Delta \Delta}^{\prime}}+a_{\epsilon+\epsilon_{\Delta}^{\prime}}
$$


Note that their dependence on the final states are understood. Using the isospin symmetry, we find

$$
\begin{aligned}
A^{(+-)} & =\sqrt{\frac{2}{3}} a_{0}+\sqrt{\frac{1}{3}} a_{2} \\
A^{(00)} & =\sqrt{\frac{1}{3}} a_{0}-\sqrt{\frac{2}{3}} a_{2}
\end{aligned}
$$

with $A^{(+-)}$and $A^{(00)}$ the amplitudes for the decay modes $K^{0}\left(\bar{K}^{0}\right) \rightarrow \pi^{+} \pi^{-}$and $K^{0}\left(\bar{K}^{0}\right) \rightarrow$ $\pi^{0} \pi^{0}$ respectively, where $a_{0}$ and $a_{2}$ correspond to the isospin $I=0$ and $I=2$ amplitudes. The same decomposition holds for $B^{(+-)}$and $B^{(00)}$ amplitudes 2 . Considering the fact that $\omega=\left|A_{2}\right| /\left|A_{0}\right| \simeq 1 / 22<<1$ due to the $\Delta I=1 / 2$ rule, we obtain

$$
\begin{aligned}
\hat{a}_{\epsilon^{\prime}}^{(+-)} & \simeq a_{\epsilon^{\prime}}+a_{\Delta \Delta}^{\prime}+\tilde{a}_{\varepsilon \Delta}+a_{\varepsilon \Delta}^{0}, \\
\hat{a}_{\epsilon^{\prime}}^{(00)} & \simeq-2 a_{\epsilon^{\prime}}-2 a_{\Delta \Delta}^{\prime}-2 \tilde{a}_{\varepsilon \Delta}+a_{\varepsilon \Delta}^{0},
\end{aligned}
$$

and

$$
\begin{aligned}
& \hat{a}_{\epsilon+\epsilon^{\prime}}^{(+-)} \simeq a_{\epsilon+\epsilon^{\prime}}^{0}+a_{\epsilon+\epsilon_{\Delta \Delta}^{\prime}}^{0}+\tilde{a}_{\epsilon+\epsilon^{\prime}}+\tilde{a}_{\epsilon+\epsilon_{\Delta \Delta}^{\prime}}+a_{\epsilon+\epsilon_{\Delta}^{\prime}} \\
& \hat{a}_{\epsilon+\epsilon^{\prime}}^{(00)} \simeq a_{\epsilon+\epsilon^{\prime}}^{0}+a_{\epsilon+\epsilon_{\Delta \Delta}^{\prime}}^{0}-2 \tilde{a}_{\epsilon+\epsilon^{\prime}}-2 \tilde{a}_{\epsilon+\epsilon_{\Delta \Delta}^{\prime}}-2 a_{\epsilon+\epsilon_{\Delta}^{\prime}}
\end{aligned}
$$

with

$$
\begin{aligned}
& a_{\varepsilon \Delta}^{0}=2 \operatorname{Re} \Delta_{0}=2 \operatorname{Re}\left(\frac{B_{0}}{A_{0}}\right), \quad \tilde{a}_{\varepsilon \Delta}=2 \operatorname{Re}\left[\frac{A_{2}}{A_{0}}\left(\Delta_{2}-\Delta_{0}\right)\right] \cos \left(\delta_{0}-\delta_{2}\right) \\
& a_{\epsilon+\epsilon^{\prime}}^{0}=2 \frac{\operatorname{Im} \epsilon_{K}}{1+\left|\epsilon_{K}\right|^{2}} \operatorname{Re}\left(\frac{A_{0}^{*}}{A_{0}}\right)+2 \frac{1-\left|\epsilon_{K}\right|^{2}}{1+\left|\epsilon_{K}\right|^{2}} \operatorname{Im}\left(\frac{A_{0}^{*}}{A_{0}}\right) \\
& \tilde{a}_{\epsilon+\epsilon^{\prime}} \simeq 4\left[\frac{\operatorname{Im} \epsilon_{K}}{1+\left|\epsilon_{K}\right|^{2}} \operatorname{Re}\left(\frac{A_{2}^{*}}{A_{0}}\right)+\frac{1-\left|\epsilon_{K}\right|^{2}}{1+\left|\epsilon_{K}\right|^{2}} \operatorname{Im}\left(\frac{A_{2}^{*}}{A_{0}}\right)\right] \cos \left(\delta_{0}-\delta_{2}\right) \\
& a_{\epsilon+\epsilon_{\Delta \Delta}^{\prime}}^{0}=-2 \frac{\operatorname{Im} \epsilon_{K}}{1+\left|\epsilon_{K}\right|^{2}} \operatorname{Re}\left(\frac{A_{0}^{*}}{A_{0}} \Delta_{0}^{* 2}\right)-2 \frac{1-\left|\epsilon_{K}\right|^{2}}{1+\left|\epsilon_{K}\right|^{2}} \operatorname{Im}\left(\frac{A_{0}^{*}}{A_{0}} \Delta_{0}^{* 2}\right) \\
& \tilde{a}_{\epsilon+\epsilon_{\Delta \Delta}^{\prime}} \simeq-4\left[\frac{\operatorname{Im} \epsilon_{K}}{1+\left|\epsilon_{K}\right|^{2}} \operatorname{Re}\left(\frac{A_{2}^{*}}{A_{0}} \Delta_{0}^{*} \Delta_{2}^{*}\right)+\frac{1-\left|\epsilon_{K}\right|^{2}}{1+\left|\epsilon_{K}\right|^{2}} \operatorname{Im}\left(\frac{A_{2}^{*}}{A_{0}} \Delta_{0}^{*} \Delta_{2}^{*}\right)\right] \cos \left(\delta_{0}-\delta_{2}\right)
\end{aligned}
$$

where we have neglected quadratic terms of $\omega=\left|A_{2} / A_{0}\right|$. Note that the above results hold for any choice of phase conventions. It is then obvious that

$$
a_{\varepsilon \Delta}^{0}=\frac{2}{3} \hat{a}_{\epsilon^{\prime}}^{(+-)}+\frac{1}{3} \hat{a}_{\epsilon^{\prime}}^{(00)}
$$

which shows that once the asymmetries $\hat{a}_{\epsilon^{\prime}}^{(+-)}$and $\hat{a}_{\epsilon^{\prime}}^{(00)}$ are measured, their combination given above will allow one to extract a clean signature of CPT violation arising from the decay amplitudes. Where the values of $\hat{a}_{\epsilon^{\prime}}^{(+-)}$and $\hat{a}_{\epsilon^{\prime}}^{(00)}$ can be simply extracted from the

\footnotetext{
${ }^{2}$ Note that normalization of $A^{(00)}$ is smaller by a factor $\sqrt{2}$ than the usual one ocuuring in literature.
} 
asymmetry $A_{C P+C P T}(t)$ at $t=0$ in eq. (39). It is noticed that when $\left|\Delta_{0}\right|<<1$, i.e., $\left|a_{\epsilon+\epsilon_{\Delta \Delta}^{\prime}}^{0}\right|<<\left|a_{\epsilon+\epsilon^{\prime}}^{0}\right|$ (while $\Delta_{2}$ could remain at the order of one), one has

$$
a_{\epsilon+\epsilon^{\prime}}^{0} \simeq \frac{2}{3} \hat{a}_{\epsilon+\epsilon^{\prime}}^{(+-)}+\frac{1}{3} \hat{a}_{\epsilon+\epsilon^{\prime}}^{(00)}
$$

which indicates that by measuring $\hat{a}_{\epsilon+\epsilon^{\prime}}^{(+-)}$and $\hat{a}_{\epsilon+\epsilon^{\prime}}^{(00)}$ one may extract the direct-indirect mixed-induced $\mathrm{CP}$ violation.

\section{Conclusions}

In summary, we have developed the general model-independent and rephase-invariant formalism for testing CP- and CPT-noninvariant observables in meson decays. The formalism presented in previous articles for CPT is based on the density matrix approach 15. In our article, we present a complete time-dependent and rephase-invariant formulation in terms of amplitudes. The rephase invariance of all CP and CPT noninvariant observables is maintained throughout the calculation. All possible independent observables have been classified systematically, which is more general and complete than the published results and can be used for all meson decays. This enables one to separately measure different types of CPT- and CP-violating observables and to neatly distinguish effects of $\mathrm{CPT}$ from $\mathrm{CP}$ violation. The formalism which involves many and elaborate definitions is directly related to fundamental parameters and can prove advantageous in establishing CPT-violating parameters from time-dependent measurements of meson decays. Several time-dependent CPT- and CP- asymmetries have been introduced, which led to some interesting observations:

i). As long as measurements of the asymmetry $A_{C P+C P T}(t)$ in the neutral meson decays (classified in the scenario i) in section 5 ) is not a constant but depends on time, one can conclude that CPT invariance is broken due to mixing;

ii). For the semileptonic decays $M^{0} \rightarrow M^{\prime}-l \nu$, one may come to a strong statement that once the asymmetry $A_{C P+C P T}(t)$ is not zero, then CPT must be violated. Among the decays the semileptonic decays are the more representative and perhaps the easiest to measure.

iii). A combined measurement of several time-dependent CPT- and CP- asymmetries from semileptonic and nonleptonic decays is necessary in order to isolate separately the indirect and direct CPT- and CP-violating effects.

Extraction of a clean signature on $\mathrm{CPT}, \mathrm{CP}$ and $\mathrm{T}$ violation will play an important role in testing the standard model and local quantum field theory and in addition provides an interesting window for probing new physics. For all these reasons, this topic attracts a lot of attention 16. We hope that the general rephase-invariant formalism presented in this paper will be useful for further studies of $\mathrm{CPT}, \mathrm{CP}$ and $\mathrm{T}$ in the neutral meson systems produced at B-factories, the $\Phi$-factory[17] and colliders. 
Acknowledgements: W.P was supported in part by the US department of Energy, Division of High Energy Physics, under Grant DOE/ER/0 1545-778. Two of us (E.A.P and Y.L.W) thanks Bundesministerium für Bildung, Wissenschaft, Forschung und Technologie (BMBF), 057D093P(7), Bonn, FRG, and DFG Antrag PA-10-1 for the financial support. Y.L.W acknowledges the support by the NSF of China under Grant 19625514. 


\section{Appendix}

Here we collect some useful formuli.

The definitions for the rephase-invariant observables:

$$
\begin{aligned}
\hat{a}_{\epsilon^{\prime}} & =\frac{1-|h / g|^{2}}{1+|h / g|^{2}}=\frac{2 R e \varepsilon_{M}^{\prime}}{1+\left|\varepsilon_{M}^{\prime}\right|^{2}}, \\
\hat{a}_{\epsilon_{S}+\epsilon^{\prime}} & =\frac{-4 \operatorname{Im}\left(q_{S} h / p_{S} g\right)}{\left(1+\left|q_{S} / p_{S}\right|^{2}\right)\left(1+|h / g|^{2}\right)} \\
& =\frac{1}{1-a_{\epsilon} a_{\Delta}}\left[\hat{a}_{\epsilon+\epsilon^{\prime}} \sqrt{1-a_{\Delta}^{2}-a_{\Delta}^{\prime 2}}-a_{\Delta}^{\prime}\left(1+\hat{a}_{\epsilon \epsilon^{\prime}}\right)\right], \quad[A 1] \\
\hat{a}_{\epsilon_{S} \epsilon^{\prime}} & =\frac{4 R e\left(q_{S} h / p_{S} g\right)}{\left(1+\left|q_{S} / p_{S}\right|^{2}\right)\left(1+|h / g|^{2}\right)}-1 \\
& =\frac{1}{1-a_{\epsilon} a_{\Delta}}\left[\hat{a}_{\epsilon \epsilon^{\prime}} \sqrt{1-a_{\Delta}^{2}-a_{\Delta}^{\prime 2}}+a_{\Delta}^{\prime} \hat{a}_{\epsilon+\epsilon^{\prime}}+\left(\sqrt{1-a_{\Delta}^{2}-a_{\Delta}^{\prime 2}}-1\right)+a_{\epsilon} a_{\Delta}\right],
\end{aligned}
$$

with

$$
\begin{aligned}
\hat{a}_{\epsilon+\epsilon^{\prime}} & =\frac{-4 \operatorname{Im}(q h / p g)}{\left(1+|q / p|^{2}\right)\left(1+|h / g|^{2}\right)}=\frac{2 \operatorname{Im} \epsilon_{M}\left(1-\left|\varepsilon_{M}^{\prime}\right|^{2}\right)+2 \operatorname{Im}_{M}^{\prime}\left(1-\left|\epsilon_{M}\right|^{2}\right)}{\left(1+\left|\epsilon_{M}\right|^{2}\right)\left(1+\left|\varepsilon_{M}^{\prime}\right|^{2}\right)}, \\
\hat{a}_{\epsilon \epsilon^{\prime}} & =\frac{4 R e(q h / p g)}{\left(1+|q / p|^{2}\right)\left(1+|h / g|^{2}\right)}-1=\frac{4 \operatorname{Im} \epsilon_{M} \operatorname{Im} \varepsilon_{M}^{\prime}-2\left(\left|\epsilon_{M}\right|^{2}+\left|\varepsilon_{M}^{\prime}\right|^{2}\right)}{\left(1+\left|\epsilon_{M}\right|^{2}\right)\left(1+\left|\varepsilon_{M}^{\prime}\right|^{2}\right)} .
\end{aligned}
$$

Rephase invariant observables for purely CP and CPT violation

$$
\begin{aligned}
a_{\epsilon^{\prime \prime}} & =\frac{\left|\sum_{i} A_{i} e^{i \delta_{i}}\right|^{2}-\left|\sum_{i} A_{i}^{*} e^{i \delta_{i}}\right|^{2}}{\left|\sum_{i} A_{i} e^{i \delta_{i}}\right|^{2}+\left|\sum_{i} A_{i}^{*} e^{i \delta_{i}}\right|^{2}}=-\frac{2 \sum_{i j} A_{i} A_{j}^{*} \sin \left(\delta_{i}-\delta_{j}\right)}{\left|\sum_{i} A_{i} e^{i \delta_{i}}\right|^{2}+\mid \sum_{i} A_{i}^{*} e^{\left.i \delta_{i}\right|^{2}}}, \\
a_{\varepsilon \Delta} & =\frac{2 \sum_{i, j} A_{i} A_{j}^{*}\left(\Delta_{i}+\Delta_{j}^{*}\right) \cos \left(\delta_{i}-\delta_{j}\right)}{\left|\sum_{i} A_{i} e^{i \delta_{i}}\right|^{2}+\left|\sum_{i} A_{i}^{*} e^{i \delta_{i}}\right|^{2}}, \\
a_{\varepsilon \Delta}^{\prime} & =\frac{2 i \sum_{i, j} A_{i} A_{j}^{*}\left(\Delta_{i}+\Delta_{j}^{*}\right) \sin \left(\delta_{i}-\delta_{j}\right)}{\left|\sum_{i} A_{i} e^{i \delta_{i}}\right|^{2}+\left|\sum_{i} A_{i}^{*} e^{i \delta_{i}}\right|^{2}}, \\
a_{\Delta \Delta} & =\frac{2 \sum_{i, j} A_{i} A_{j}^{*} \Delta_{i} \Delta_{j}^{*} \cos \left(\delta_{i}-\delta_{j}\right.}{\left|\sum_{i} A_{i} e^{i \delta_{i}}\right|^{2}+\left|\sum_{i} A_{i}^{*} e^{i \delta_{i}}\right|^{2}} \\
a_{\Delta \Delta}^{\prime} & =\frac{2 i \sum_{i, j} A_{i} A_{j}^{*} \Delta_{i} \Delta_{j}^{*} \sin \left(\delta_{i}-\delta_{j}\right)}{\left|\sum_{i} A_{i} e^{i \delta_{i}}\right|^{2}+\left|\sum_{i} A_{i}^{*} e^{i \delta_{i}}\right|^{2}}
\end{aligned}
$$

with $\Delta_{i}=B_{i} / A_{i}$. Here $\Delta_{i}$ are rephase-invariant quantities and characterize direct CPT violation in the decay amplitudes.

$$
\begin{aligned}
a_{\epsilon+\epsilon^{\prime}} & =\frac{2 \operatorname{Im} \epsilon_{M}\left(1-\left|\epsilon_{M}^{\prime}\right|^{2}\right)+2 \operatorname{Im} \epsilon_{M}^{\prime}\left(1-\left|\epsilon_{M}\right|^{2}\right)}{\left(1+\left|\epsilon_{M}\right|^{2}\right)\left(1+\left|\epsilon_{M}^{\prime}\right|^{2}\right)} \\
a_{\epsilon+\epsilon_{\Delta}^{\prime}} & =\frac{2 \operatorname{Im} \epsilon_{M}\left(1-\left|\epsilon_{\Delta}^{\prime}\right|^{2}\right)+2 \operatorname{Im} \epsilon_{\Delta}^{\prime}\left(1-\left|\epsilon_{M}\right|^{2}\right)}{\left(1+\left|\epsilon_{M}\right|^{2}\right)\left(1+\left|\epsilon_{\Delta}^{\prime}\right|^{2}\right)}
\end{aligned}
$$




$$
a_{\epsilon+\epsilon_{\Delta \Delta}^{\prime}}=\frac{2 \operatorname{Im} \epsilon_{M}\left(1-\left|\epsilon_{\Delta \Delta}^{\prime}\right|^{2}\right)+2 \operatorname{Im} \epsilon_{\Delta \Delta}^{\prime}\left(1-\left|\epsilon_{M}\right|^{2}\right)}{\left(1+\left|\epsilon_{M}\right|^{2}\right)\left(1+\left|\epsilon_{\Delta \Delta}^{\prime}\right|^{2}\right)}
$$

with

$$
\begin{aligned}
& \frac{1-\left|\epsilon_{M}^{\prime}\right|^{2}}{1+\left|\epsilon_{M}^{\prime}\right|^{2}}=\frac{2 \sum_{i, j} \operatorname{Re}\left(A_{i} A_{j}\right) \cos \left(\delta_{i}-\delta_{j}\right)}{\left|\sum_{i} A_{i} e^{i \delta_{i}}\right|^{2}+\left|\sum_{i} A_{i}^{*} e^{i \delta_{i}}\right|^{2}}, \\
& \frac{1-\left|\epsilon_{\Delta}^{\prime}\right|^{2}}{1+\left|\epsilon_{\Delta}^{\prime}\right|^{2}}=-\frac{2 \sum_{i, j} \operatorname{Im}\left[A_{i} A_{j}\left(\Delta_{i}-\Delta_{j}\right)\right] \sin \left(\delta_{i}-\delta_{j}\right)}{\left|\sum_{i} A_{i} e^{i \delta_{i}}\right|^{2}+\left|\sum_{i} A_{i}^{*} e^{i \delta_{i}}\right|^{2}}, \\
& \frac{1-\left|\epsilon_{\Delta \Delta}^{\prime}\right|^{2}}{1+\left|\epsilon_{\Delta \Delta}^{\prime}\right|^{2}}=-\frac{2 \sum_{i, j} \operatorname{Re}\left[A_{i} A_{j}\left(\Delta_{i} \Delta_{j}\right)\right] \cos \left(\delta_{i}-\delta_{j}\right)}{\left|\sum_{i} A_{i} e^{i \delta_{i}}\right|^{2}+\left|\sum_{i} A_{i}^{*} e^{i \delta_{i}}\right|^{2}}, \\
& \frac{2 I m \epsilon_{M}^{\prime}}{1+\left|\epsilon_{M}^{\prime}\right|^{2}}=-\frac{2 \sum_{i, j} \operatorname{Im}\left(A_{i} A_{j}\right) \cos \left(\delta_{i}-\delta_{j}\right)}{\left|\sum_{i} A_{i} e^{i \delta_{i}}\right|^{2}+\left|\sum_{i} A_{i}^{*} e^{i \delta_{i}}\right|^{2}},
\end{aligned}
$$

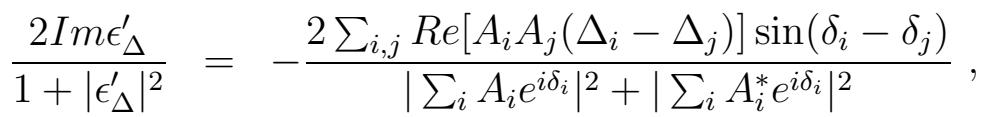

$$
\begin{aligned}
& \frac{2 \operatorname{Im} \epsilon_{\Delta \Delta}^{\prime}}{1+\left|\epsilon_{\Delta \Delta}^{\prime}\right|^{2}}=\frac{2 \sum_{i, j} \operatorname{Im}\left[A_{i} A_{j}\left(\Delta_{i} \Delta_{j}\right)\right] \cos \left(\delta_{i}-\delta_{j}\right)}{\left|\sum_{i} A_{i} e^{i \delta_{i}}\right|^{2}+\left|\sum_{i} A_{i}^{*} e^{i \delta_{i}}\right|^{2}} .
\end{aligned}
$$

The exact expressions for the time-dependent CP and CPT asymmetries in the scenario i):

$$
\begin{aligned}
& A_{C P+C P T}(t)=\frac{\Gamma\left(M^{0}(t) \rightarrow f\right)-\Gamma\left(\bar{M}^{0}(t) \rightarrow \bar{f}\right)}{\Gamma\left(M^{0}(t) \rightarrow f\right)+\Gamma\left(\bar{M}^{0}(t) \rightarrow \bar{f}\right)}, \\
& =\frac{\hat{a}_{\epsilon^{\prime \prime}}+2 \mathcal{A}_{C P T}(t) /\left[\left(1+\left|\eta_{\Delta}\right|^{2}\right) \cosh \Delta \Gamma t+\left(1-\left|\eta_{\Delta}\right|^{2}\right) \cos \Delta m t\right]}{1+2 \hat{a}_{\epsilon^{\prime \prime}} \mathcal{A}_{C P T}(t) /\left[\left(1+\left|\eta_{\Delta}\right|^{2}\right) \cosh \Delta \Gamma t+\left(1-\left|\eta_{\Delta}\right|^{2}\right) \cos \Delta m t\right]}, \\
& A_{C P+C P T}^{\prime}(t)=\frac{\Gamma\left(\bar{M}^{0}(t) \rightarrow f\right)-\Gamma\left(M^{0}(t) \rightarrow \bar{f}\right)}{\Gamma\left(\bar{M}^{0}(t) \rightarrow f\right)+\Gamma\left(M^{0}(t) \rightarrow \bar{f}\right)}, \\
& =\left(\hat{a}_{\epsilon^{\prime \prime}}+\frac{2 a_{\epsilon}}{1+a_{\epsilon}^{2}}\right) /\left(1+\frac{2 a_{\epsilon}}{1+a_{\epsilon}^{2}} \hat{a}_{\epsilon^{\prime \prime}}\right) \text {, } \\
& A_{C P+C P T}^{\prime \prime}(t)=\frac{\Gamma\left(M^{0}(t) \rightarrow f\right)-\Gamma\left(\bar{M}^{0}(t) \rightarrow f\right)}{\Gamma\left(M^{0}(t) \rightarrow f\right)+\Gamma\left(\bar{M}^{0}(t) \rightarrow f\right)}, \\
& =\frac{\frac{1-a_{\Delta}^{2}-a_{\Delta}^{\prime 2}+a_{\epsilon} a_{\Delta}^{\prime 2}}{\left(1-a_{\epsilon}\right)\left(1-a_{\Delta}^{\prime 2}\right)} \cos \Delta m t-\frac{a_{\epsilon}-a_{\Delta}^{2}}{\left(1-a_{\epsilon}\right)\left(1-a_{\Delta}^{\prime 2}\right)} \cosh \Delta \Gamma t+\mathcal{A}_{C P T}(t)}{\frac{1-a_{\epsilon} a_{\Delta}^{2}}{\left(1-a_{\epsilon}\right)\left(1-a_{\Delta}^{\prime 2}\right)} \cosh \Delta \Gamma t-\frac{a_{\epsilon}\left(1-a_{\Delta}^{2}-a_{\Delta}^{\prime 2}\right)+a_{\Delta}^{\prime 2}}{\left(1-a_{\epsilon}\right)\left(1-a_{\Delta}^{\prime 2}\right)} \cos \Delta m t+\mathcal{A}_{C P T}(t)},
\end{aligned}
$$

with

$$
\mathcal{A}_{C P T}=-\frac{a_{\Delta}}{1-a_{\Delta}^{\prime 2}} \sinh \Delta \Gamma t+\frac{a_{\Delta}^{\prime} \sqrt{1-a_{\Delta}^{2}-a_{\Delta}^{\prime 2}}}{1-a_{\Delta}^{\prime 2}} \sin \Delta m t,
$$

and in the scenario ii): 


$$
A_{C P+C P T}(t)=\frac{\hat{\Delta}_{m}(t)+\Delta_{C P T}(t)-a_{\epsilon_{S}}\left(\hat{\Delta}_{\gamma}(t)+\Delta_{C P T}^{\prime}(t)\right)}{\hat{\Delta}_{\gamma}(t)+\Delta_{C P T}^{\prime}(t)-a_{\epsilon_{S}}\left(\hat{\Delta}_{m}(t)+\Delta_{C P T}(t)\right)}
$$

with

$$
\begin{aligned}
& \hat{\Delta}_{m}(t)=\left(a_{\epsilon_{S}}+\hat{a}_{\epsilon^{\prime}}\right) \cos (\Delta m t)+\hat{a}_{\epsilon_{S}+\epsilon^{\prime}} \sin (\Delta m t) \\
& \hat{\Delta}_{\gamma}(t)=\left(1+a_{\epsilon_{S}} \hat{a}_{\epsilon^{\prime}}\right) \cosh (\Delta \Gamma t)+\left(1+\hat{a}_{\epsilon \epsilon^{\prime}}\right) \sinh (\Delta \Gamma t),
\end{aligned}
$$

and

$$
\begin{aligned}
\Delta_{C P T}(t) & =\left(2+a_{\epsilon_{S}} \hat{a}_{\epsilon^{\prime}}+\hat{a}_{\epsilon_{S \epsilon^{\prime}}}\right)\left[\frac{a_{\Delta}}{1-a_{\Delta}^{\prime 2}}\left(\cos \Delta m t-e^{\Delta \Gamma t}\right)+\frac{a_{\Delta}^{\prime}}{1-a_{\Delta}^{\prime 2}} \sin \Delta m t\right] \\
\Delta_{C P T}^{\prime}(t) & =-\left[\frac{a_{\Delta}}{1-a_{\Delta}^{\prime 2}}\left(a_{\epsilon_{S}}+\hat{a}_{\epsilon^{\prime}}\right)+\frac{a_{\Delta}^{\prime}}{1-a_{\Delta}^{\prime 2}} \hat{a}_{\epsilon_{S}+\epsilon^{\prime}}\right]\left(\cos \Delta m t-e^{-\Delta \Gamma t}\right) \\
& +\left[\frac{a_{\Delta}^{\prime}}{1-a_{\Delta}^{\prime 2}}\left(a_{\epsilon_{S}}+\hat{a}_{\epsilon^{\prime}}\right)-\frac{a_{\Delta}}{1-a_{\Delta}^{\prime 2}} \hat{a}_{\epsilon_{S}+\epsilon^{\prime}}\right] \sin \Delta m t, \quad[A 12] \\
& -\left(2+a_{\epsilon_{S}} \hat{a}_{\epsilon^{\prime}}+\hat{a}_{\epsilon_{S} \epsilon^{\prime}}\right) \frac{a_{\Delta}^{2}+a_{\Delta}^{\prime 2}}{\left(1-a_{\Delta}^{\prime 2}\right)^{2}}(\cos \Delta m t-\cosh \Delta \Gamma t)
\end{aligned}
$$

Note that when CPT is conserved, $\Delta_{C P T}(t)=\Delta_{C P T}^{\prime}=0$. 


\section{References}

[1] T.D. Lee and C.N. Yang, Phys. Rev. 104, 254 (1956).

[2] C.S. Wu, E. Ambler, R.W. Hayward, D. Hoppes and R.P. Hudson, Phys. Rev. 105, 1413 (1957).

[3] R.L. Garwin, L.M. Lederman and M. Weinrich, Phys. Rev. 105, 1415 (1957).

[4] J.I. Friedman and V.L. Telegdi, Phys. Rev. 105, 1681 (1957).

[5] R. Christenson, J. Cronin, V.L. Fitch and R. Turlay, Phys. Rev. Lett. 13, 138 (1964).

[6] J. Schwinger, Phys. Rev. 82, 914 (1951); G. Lueders, Dansk. Mat. Fys. Medd. 28, 17 (1954); W. Pauli, Niels Bohr and the Development of Physics (Pergamon, New York, 1955).

[7] For recent analyses see for example: C.O. Dib and R.D. Peccei, Phys. Rev. D46, 2265 (1992); C.D. Buchanan, et al., Phys. Rev. D45, 4088 (1992).

[8] J.S. Bell and J. Steinberger, Proc. Oxford Int. Conf. on elementary particles, 1965, p. 195.

[9] J.W. Cronin, Rev. Mod. Phys. 53, 373 (1981).

[10] V.V. Barmin et al., Nucl. Phys. B247, 293 (1984).

[11] W.F. Palmer and Y.L. Wu, Phys. Lett. B350, 245 (1995).

[12] see for example, L. Lavoura, Ann. Phys. 207428 (1991), and references therein.

[13] T.D. Lee and C.S. Wu, Annu. Rev. Nucl. Sci. 16, 471 (1966).

[14] E.A. Paschos and R. Zacher, Z. Phys. C28 521 (1985);

For a review see for example, E.A. Paschos and U. Türke, Phys. Rep. 178147 (1989).

[15] J. Ellis, J.S. Hagelin, D.V. Nanopoulos and M. Srednichi, Nucl. Phys. B241, 381 (1984);

P. Huet and M. E. Peskin, Nucl. Phys. B434, 3 (1995).

J. Ellis, J.L. Lopez, N.E. Mavromatos and D.V. Nanopoulos, Phys. Rev. D 53, 3846 (1996).

[16] L. Wolfenstein, Phys.Rev.Lett. 83911 (1999);

L. Lavoura, hep-ph/9911209;

A. I. Sanda, hep-ph/9902353;

L. Lavoura, J. P. Silva, hep-ph/9902348;

P. Huet, hep-ph/9607435;

J. Ellis, N.E. Mavromatos, D.V. Nanopoulos, hep-ph/9607434;

R. Adler, et al (CPLEAR Collaboration), J. Ellis, J. Lopez, N. Mavromatos, D. Nanopoulos, hep-ex/9511001; 
N. Mavromatos, T. Ruf (for the collaboration: J. Ellis, J. Lopez, N. Mavromatos, D. Nanopoulos, and the CPLEAR Collaboration), hep-ph/9506395.

[17] See for example, L. Maiani, in 'The Second DA $\Phi$ NE Physics Handbook', edited by L. Maiani, G. Pancheri and N. Paver, p.3-26; G. D'Ambrosio, G. Isidoni and A. Pugliese, ibid., p.63-95; and references therein. 